\title{
Endothelin-1 regulates cardiac sympathetic innervation in the rodent heart by controlling nerve growth factor expression
}

\author{
Masaki leda, ${ }^{1}$ Keiichi Fukuda,, ${ }^{12}$ Yasuyo Hisaka, ${ }^{2}$ Kensuke Kimura, ${ }^{1}$ Haruko Kawaguchi, ${ }^{2}$ Jun Fujita, ${ }^{1}$ \\ Kouji Shimoda, ${ }^{3}$ Eiko Takeshita, ${ }^{4}$ Hideyuki Okano, ${ }^{5}$ Yukiko Kurihara, ${ }^{6}$ Hiroki Kurihara, ${ }^{6}$ Junji Ishida, ${ }^{7}$ \\ Akiyoshi Fukamizu,7 Howard J. Federoff, ${ }^{7}$ and Satoshi Ogawa1 \\ Cardiopulmonary Division, Department of Internal Medicine, ${ }^{2}$ Institute for Advanced Cardiac Therapeutics, ${ }^{3}$ Laboratory Animal Center, \\ ${ }^{4}$ Department of Laboratory Medicine, and ${ }^{5}$ Department of Physiology, Keio University School of Medicine, Tokyo, Japan. \\ ${ }^{6}$ Division of Physiological Chemistry and Metabolism, Department of Biochemistry and Molecular Biology, Faculty of Medicine, University of Tokyo, Tokyo, Japan. \\ ${ }^{7}$ Center for Tsukuba Advanced Research Alliance and Institute of Applied Biochemistry, University of Tsukuba, Ibaraki, Japan. \\ ¿University of Rochester Medical Center, Center for Aging and Developmental Biology, Rochester, New York, USA.
}

\begin{abstract}
The cardiac sympathetic nerve plays an important role in regulating cardiac function, and nerve growth factor (NGF) contributes to its development and maintenance. However, little is known about the molecular mechanisms that regulate NGF expression and sympathetic innervation of the heart. In an effort to identify regulators of NGF in cardiomyocytes, we found that endothelin-1 specifically upregulated NGF expression in primary cultured cardiomyocytes. Endothelin-1-induced NGF augmentation was mediated by the endothelin-A receptor, Gi $\beta \gamma$, PKC, the Src family, EGFR, extracellular signal-regulated kinase, p38MAPK, activator protein-1, and the CCAAT/enhancer-binding protein $\delta$ element. Either conditioned medium or coculture with endothelin-1-stimulated cardiomyocytes caused NGF-mediated PC12 cell differentiation. NGF expression, cardiac sympathetic innervation, and norepinephrine concentration were specifically reduced in endothelin-1deficient mouse hearts, but not in angiotensinogen-deficient mice. In endothelin-1-deficient mice the sympathetic stellate ganglia exhibited excess apoptosis and displayed loss of neurons at the late embryonic stage. Furthermore, cardiac-specific overexpression of NGF in endothelin-1-deficient mice overcame the reduced sympathetic innervation and loss of stellate ganglia neurons. These findings indicate that endothelin-1 regulates NGF expression in cardiomyocytes and plays a critical role in sympathetic innervation of the heart.
\end{abstract}

\section{Introduction}

Cardiac tissues are extensively innervated by autonomic nerves. The cardiac sympathetic nerve plays an important role in modulating heart rate, conduction velocity, myocardial contraction, and relaxation. Although several molecules that regulate the development of the heart have been well characterized, little is known about the mechanism that regulates sympathetic innervation of the heart. The cardiac sympathetic nerve extends from the sympathetic neuron in stellate ganglia (SG), which is derived from the neural crest (1). Nerve growth factor (NGF) is a prototypic member of the neurotrophin family, members of which are critical for the differentiation, survival, and synaptic activity of the peripheral sympathetic and sensory nervous systems $(2,3)$. Levels of NGF expression within innervated tissues roughly correspond to innervation density (4). The volume of

Nonstandard abbreviations used: activator protein-1 (AP-1); angiotensinogendeficient (mouse) $\left(\mathrm{Atg}^{-/}\right)$; B-type natriuretic peptide (BNP); carboxyl terminus of $\beta$-adrenergic receptor kinase ( $\beta$ ARK-ct); cardiac-specific overexpression of $N G F$ gene under the control of the cardiac-specific $\alpha$-myosin heavy chain promoter (mouse) (MHC-NGF); CCAAT/enhancer-binding protein $\delta$ (C/EBPס); C-terminal Src kinase (Csk); deletion mutant of EGFR (533delEGFR); dominant-negative mutant adenovirus of extracellular signal-regulated kinase (DN-ERK); dominant-negative mutant adenovirus of p38MAPK (DN-p38); embryonic day (E); endothelin-1 (ET-1); endothelin-A (ET $\mathrm{A}_{\mathrm{A}}$; ET-1-deficient (mouse) $\left(\mathrm{Edn1^{-/ }}\right)$; extracellular signal-regulated kinase (ERK); growth-associated protein 43 (GAP43); leukemia inhibitory factor (LIF); nerve growth factor (NGF); pertussis toxin (PTX); protein gene product 9.5 (PGP9.5); protein kinase A (PKA); stellate ganglia (SG); tyrosine hydroxylase (TH).

Conflict of interest: The authors have declared that no conflict of interest exists. Citation for this article: J. Clin. Invest. 113:876-884 (2004). doi:10.1172/JCI200419480. sympathetic ganglion is reduced by at least $80 \%$ at postnatal day 3 in mice with a disruption of the NGF gene. In mice that lack the NGF receptor TrkA, no neurons remain at postnatal day 9 (2). Deletion of a single copy of the NGF gene results in a $50 \%$ reduction in sympathetic neurons (5), while overexpression of NGF in the heart results in cardiac hyperinnervation and hyperplasia in SG neurons (6). These results demonstrate the importance of NGF in the regulation of sympathetic neuron development and innervation.

In pathological states, NGF production in the heart is variable. In ischemic hearts, an increase in cardiac NGF leads to regeneration of sympathetic nerves $(7,8)$. In a previous experiment, we found that NGF was upregulated in streptozotocin-induced diabetic murine hearts (9). In contrast, it was reported that NGF and sympathetic innervation were reduced in congestive heart failure (10). Despite their importance, the molecular mechanisms that regulate NGF expression and sympathetic innervation in the heart remain poorly understood.

Endothelin-1 (ET-1) is believed to play a critical role in the pathogenesis of cardiac hypertrophy, hypertension, and atherosclerosis. Gene targeting of ET- 1 and its receptor endothelin-A $\left(\mathrm{ET}_{\mathrm{A}}\right)$ resulted in unexpected craniofacial and cardiovascular abnormalities. These phenotypes are consistent with interference of neural crest differentiation. The influence of ET-1 on neural crest development remains undetermined (11-13).

We hypothesized that ET-1 could affect the induction of neurotrophic factors, and that its disruption might contribute to the immature development of neural crest-derived cells. In this study, we found ET-1-specific induction of NGF in cardiomyocytes, iden- 
tified the signaling pathways involved, and studied the ET-1-NGF pathway-mediated development of the sympathetic nervous system in the heart. In ET-1-deficient $\left(E d n 1^{-/-}\right)$mice, the sympathetic nerve density and norepinephrine concentration were markedly reduced, and a loss of SG neurons by excess apoptosis was observed due to downregulation of cardiac NGF. Moreover, we demonstrated that the sympathetic nerve retardation was restored in $E d n 1^{-/-}$mice, which overexpressed the NGF gene under the transcriptional control of the cardiac-specific $\alpha$-myosin heavy chain promoter $\left(E d n 1^{-/} / \mathrm{MHC}-\mathrm{NGF}\right.$ mice). These findings show that ET-1 is a key regulator of NGF expression in cardiomyocytes, and that the ET-1-NGF pathway is critical for sympathetic innervation in the heart.

\section{Methods}

Cell culture. Primary culture for cardiomyocytes and cardiac fibroblasts and the cultures for PC12 cells were as described previously $(14,15)$.

RNA extraction, Northern blot, and quantitative RT-PCR. RNA and quantitative RT-PCR were performed as described previously (14). To detect the four murine NGF alternatively spliced transcripts, four primer sets were designed as follows: the exon 2-specific forward primer (for transcript a) was $5^{\prime}$-CTCCTAGTGAAGATGCTGTGCC$3^{\prime}$, the forward primer specific to exons $1 \mathrm{~B}-3 \mathrm{~B}$ (for transcript $\mathrm{b}$ ) was $5^{\prime}$-AGCGCATCGAGTTTTGGCCTGT-3', the exon 1A-specific forward primer (for transcript c) was $5^{\prime}$-TGGCTTTTCCTGGCTATGTCC-3' ${ }^{\prime}$, the exon 3A-specific forward primer (for transcript d) was $5^{\prime}$-AGTGCTTGCCTTATTGGGAC-3', and the same reverse primer was used in four primer sets, $5^{\prime}$-CTGTGGCTGTGGTCTTATCTC-3'. For Northern blot analysis, $2 \mu \mathrm{g}$ of poly $(\mathrm{A})^{+} \mathrm{RNA}$ was used. Rat NGF, B-type natriuretic peptide (BNP), and GAPDH cDNA were obtained by RT-PCR from the heart. Rat NGF primers were 5'-GCAGACCCGCAACATCACTG-3' and 5'-TCTCCAACCCACACACTGACA-3'. The primers and probes for murine NGF were forward, $5^{\prime}$-GCCAAGGACGCAGCTTTCTA-3'; reverse, 5'-GCCTGTACGCCGATCAAAA3'; and probe, 5'-FAM-CCGCAGTGAGGTGCATAGCGTA ATGTCTAMRA-3'. Primers for NGF expression in $E d n 1^{-/-} / \mathrm{MHC}-\mathrm{NGF}$ mice were forward, 5'-GATCGGCGTACAGGCAGAA-3'; reverse, 5'TGGGCTTCAGGGACAGAGTCT-3'; and probe, 5'-FAM-CGTACACAGATAGCAATG-MGB-3'. Primers for neurotrophin-3 were forward, 5'-AACATAAGAGTCACCGAGGAGAGTACT-3'; reverse, 5'ATGTC AATGGCTGAGGACTTGTC-3'; and probe, 5'-FAM-CACCCACAGGCTCTCACTGT CACACA-TAMRA-3'. The mRNA levels were normalized by comparison to GAPDH mRNA.

Reagents. Reagents were supplied by the following sources: SigmaAldrich (St. Louis, Missouri, USA) supplied ET-1 $\left(10^{-7} \mathrm{M}\right)$, angiotensin II $\left(10^{-7} \mathrm{M}\right)$, phenylephrine $\left(10^{-5} \mathrm{M}\right)$, BQ123 $\left(10^{-5} \mathrm{M}\right)$, H89 $\left(2 \times 10^{-6} \mathrm{M}\right)$, PD98059 $\left(5 \times 10^{-5} \mathrm{M}\right), \mathrm{SB} 203580\left(10^{-5} \mathrm{M}\right), \mathrm{PMA}\left(10^{-6}\right.$ $\mathrm{M})$, chelerythrine $\left(10^{-5} \mathrm{M}\right), \mathrm{IGF}-1(10 \mathrm{ng} / \mathrm{ml})$, pertussis toxin (PTX; 100 $\mathrm{ng} / \mathrm{ml})$, wortmannin $\left(10^{-8} \mathrm{M}\right), \mathrm{KN} 62\left(10^{-5} \mathrm{M}\right)$, EGTA $\left(4 \times 10^{-3} \mathrm{M}\right), 1,2$ bis(2-aminophenoxy) ethane-N,N,N $\mathrm{N}^{\prime} \mathrm{N}^{\prime}$-tetraacetic acid tetrakis (acetoxymethyl ester) (BAPTA-AM) $\left(10^{-4} \mathrm{M}\right)$, and anti-NGF blocking antibody $(1: 10,000)$. Calbiochem-Novabiochem Corp. (San Diego, California, USA) supplied PP2 $\left(10^{-5} \mathrm{M}\right)$ and AG1478 $\left(5 \times 10^{-7}\right.$ M). Santa Cruz Biotechnology Inc. (Santa Cruz, California, USA) supplied leukemia inhibitory factor (LIF; $1,000 \mathrm{U} / \mathrm{ml}$ ). Takeda Chemical Industries Ltd. (Osaka, Japan) supplied TAK044 (10-6 M).

Adenoviruses. $\beta-\mathrm{Gal}(\mathrm{LacZ})$ and the recombinant adenovirus for the carboxyl terminus of $\beta$-adrenergic receptor kinase ( $\beta$ ARK-ct) were provided by H. Kurose (University of Tokyo, Tokyo, Japan). Dominant-negative mutant adenoviruses of extracellular signal-regulated kinase (DN-ERK) and p38MAPK (DN-p38) were provided by S.
Mitsuyama (Kumamoto University, Kumamoto, Japan). Infection was performed as described previously $(16,17)$.

Preparation of conditioned medium. Cardiomyocytes were incubated in medium containing serum for 24 hours. The medium was replaced with fresh serum-free medium, and then the cells were stimulated with several factors. After 6 hours, the conditioned medium was collected and assayed.

ELISA for NGF. An ELISA kit was purchased from Promega Corp. (Madison, Wisconsin, USA).

Plasmids and luciferase assay. Luciferase plasmids containing various lengths of NGF promoter were provided by P.F. Johnson (National Cancer Institute-Frederick Cancer Research and Development Center, Frederick, Maryland, USA) (18). C-terminal Src kinase (Csk) and the deletion mutant of EGFR (533delEGFR) were provided by H. Sabe (Kyoto University, Kyoto, Japan) and H. Matsubara (Kyoto Prefectural University of Medicine, Kyoto, Japan) (19).

Differentiation of PC12 cells. Cells were examined under a phase-contrast microscope. The number of differentiated cells was counted in six randomly selected fields. The criterion for distinguishing differentiated from undifferentiated cells was neurite outgrowth greater in length than two cell-body diameters.

Coculture of PC12 cells with cardiomyocytes. PC12 cells $\left(4 \times 10^{3} / \mathrm{cm}^{2}\right)$ transfected with adenoviral LacZ were overlaid onto the cardiomyocytes $\left(4 \times 10^{4} / \mathrm{cm}^{2}\right)$. Cells were fixed and stained with X-gal reagent.

Animals. Edn1 $1^{-/}$, angiotensinogen-deficient $\left(\mathrm{Atg}^{-/}\right)$, and MHC-NGF mice were generated as described previously $(6,11,20)$. Osmotic minipumps containing BQ123 or candesartan were implanted in the pregnant $E d n 1^{+/-}$or $\mathrm{Atg}^{+/-}$mice as described previously $(12,21) . E d n 1^{+/-}$ mice were crossed with MHC-NGF mice to generate $E d n 1^{+/-} / \mathrm{MHC}$ NGF mice, which were in turn crossed with $E d n 1^{+/-}$to generate Edn1-/-/MHC-NGF mice. The Keio University Ethics Committee for Animal Experiments approved all experiments in this study.

Norepinephrine measurement. Norepinephrine concentration was determined by HPLC as described previously (22).

Immunohistochemistry in hearts. To detect nerve fibers in hearts, paraffin-embedded sections were incubated with antibody against tyrosine hydroxylase (TH; Chemicon International Inc., Temecula, California, USA), growth-associated protein 43 (GAP43; Chemicon International Inc.), and protein gene product 9.5 (PGP9.5; Cambridge Bioscience, Cambridge, United Kingdom). Following hybridization with the secondary antibody, sections were incubated with diaminobenzidine. Nerve density was determined using NIH Image, as described previously $(7,8)$.

Histology in sympathetic ganglia. For whole-mount immunostaining, embryos were fixed with paraformaldehyde and stained with anti-TH antibody as described previously (23). Paraffin-embedded sections were stained with cresyl violet, antibody against TH and Ki-67 (DAKO Corp., Carpinteria, California, USA); neuronal cell apoptosis was detected using ApopTag kit (Chemicon International Inc.) according to the manufacturer's instructions. The total number of neurons and the neuron area were determined as described previously (24).

Statistical analysis. Values are presented as means \pm SEM. Differences between groups were examined for statistical significance using Student's $t$ test or ANOVA with Fisher's protected least significant difference test. $P$ values less than 0.05 were regarded as significant.

\section{Results}

ET-1, but not angiotensin II, phenylephrine, LIF, or IGF-1, increases NGF expression in cardiomyocytes. Transcription of the NGF gene results in four different sizes by alternative splicing (25). The levels of the four 


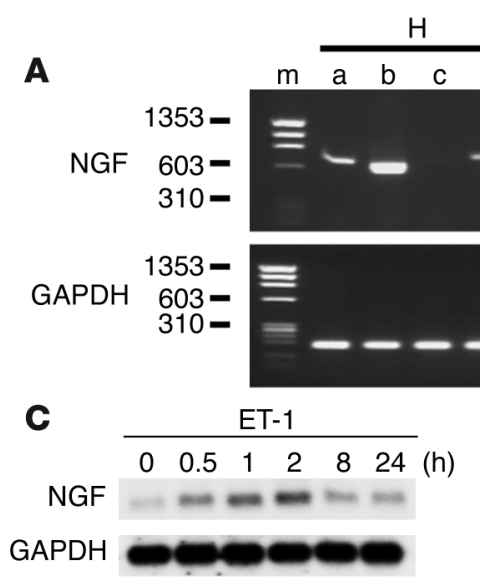

$\mathbf{F}$

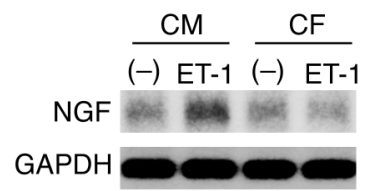

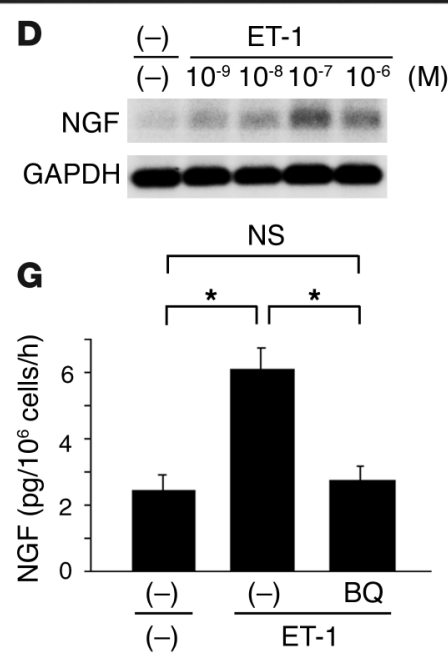

B

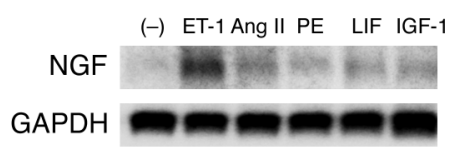

$\mathbf{E}$
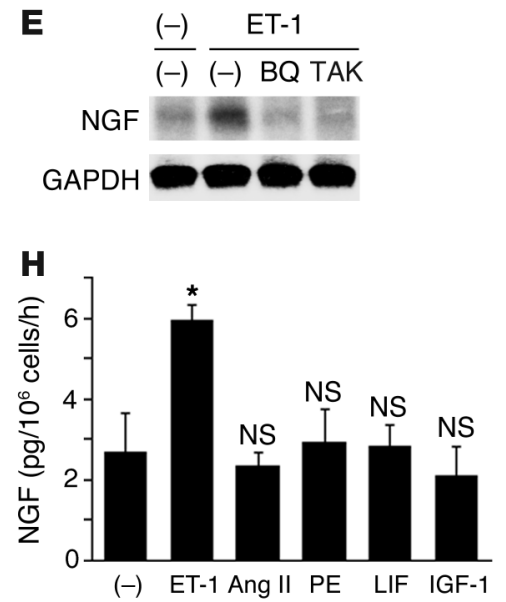

Figure 1

Specific augmentation of NGF expression by ET-1 in cardiomyocytes. (A) Gene expression of four NGF alternatively spliced transcripts (a-d) in murine heart $(\mathrm{H})$, brain $(\mathrm{BR})$, and submaxillary gland $(\mathrm{S})$ was determined by RT-PCR. The number of PCR cycles is $35 . \mathrm{m}, \mathrm{marker}$. (B) Cardiomyocytes were stimulated with ET-1, angiotensin II (Ang II), phenylephrine (PE), LIF, or IGF-1 for 2 hours. Northern blot analysis for NGF was performed. (C) Stimulation of cardiomyocytes with ET-1 for specified time intervals. (D) Stimulation of cardiomyocytes with various concentrations of ET-1. (E) Cardiomyocytes were pretreated with BQ123 (BQ) or TAK044 (TAK) for 30 minutes; then RNA was isolated 2 hours after ET-1 stimulation. (F) Induction of NGF expression by ET-1 in cardiomyocytes (CM), but not in cardiac fibroblasts (CF). Results similar to those shown in $\mathbf{A}-\mathbf{F}$ were obtained in four separate experiments. (G) NGF protein levels in conditioned medium. Augmentation of NGF protein was inhibited by pretreatment with BQ123. $(n=4$.) $(\mathbf{H})$ Secretion of NGF by cardiomyocytes was not induced by angiotensin II, phenylephrine, LIF, or IGF-1 $(n=4)$. ${ }^{*}<0.001$. NS, not significant vs. control.

NGF transcripts in the murine heart, brain, and submaxillary gland were determined by RT-PCR using the four primer sets to distinguish each transcript (Figure 1A). All transcripts were detected in the heart. Consistent with a previous study (25), transcript b was the major NGF mRNA species in the heart. Cardiomyocytes were stimulated with various cardiac hypertrophic factors, and NGF expression was ascertained by Northern blot analysis (Figure 1B). Of these factors, only ET-1 augmented NGF expression, which was induced by a 30-minute incubation and peaked after 2 hours in a dose-dependent manner (Figure 1, C and D). Preincubation with BQ123 (an $\mathrm{ET}_{\mathrm{A}}$ receptor antagonist) and TAK044 (an $\mathrm{ET}_{\mathrm{A} / \mathrm{B}}$ receptor antagonist) completely inhibited ET-1-induced NGF expression (Figure $1 \mathrm{E})$, indicating that $\mathrm{ET}_{\mathrm{A}}$ mediates this induction. To determine the cell type responsible for NGF induction, cardiomyocytes and cardiac fibroblasts were prepared separately (14), and the induction experiments were repeated. We found that NGF induction occurred only in cardiomyocytes (Figure 1F), indicating that the induction process occurs in a cell type-specific manner.

The NGF protein in ET-1-stimulated cardiomyocyte-conditioned medium increased 2.2-fold. BQ123 completely inhibited this augmentation (Figure 1G). Other hypertrophic factors had no effect on NGF expression (Figure 1H).
Intracellular signaling of ET-1-induced NGF angmentation is distinct from the ET-1-induced BNP pathway. The $\mathrm{ET}_{\mathrm{A}}$ receptor binds to three types of $\mathrm{G}$ proteins: $\mathrm{Gs}, \mathrm{Gq}$, and $\mathrm{Gi}(26)$. To determine the specific $G$ protein subtype, we pretreated the cells with the Gi inhibitor PTX or the protein kinase A (PKA) inhibitor H89. Contrary to the well-known hypertrophic-signaling pathways, NGF induction was completely repressed by PTX (Figure 2A). To determine whether Gi $\alpha$ or Gi $\beta \gamma$ is involved in this process, we transfected the cells with the $\beta A R K-c t$ adenovirus and stimulated them with ET-1. $\beta A R K-c t$ significantly attenuated induction of NGF (Figure 2B),

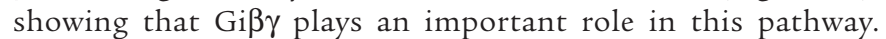
Expression of the hypertrophic markers atrial natriuretic peptide (data not shown) and BNP was unaffected in these experiments (Figure 2, A and B).

The Gi $\beta \gamma$-mediated pathway is known to activate various signaling molecules in other cell types $(16,27)$. We examined the effects of various signal transduction inhibitors and found that ET-1-induced NGF augmentation was attenuated by chelerythrine, PMA (long pretreatment), PP2, AG1478, PD98059, and SB203580, but unaffected by wortmannin or KN62 (Figure 2, D-G). Transient stimulation with PMA also induced NGF expression (Figure 2C). BNP was downregulated with PD98059 pretreatment but was unaffected by other 
A

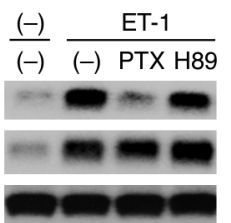

GAPDH

E $\frac{(-)}{(-)} \frac{\text { ET-1 }}{(-) \text { PD AG SB WM }}$

NGF 10 매
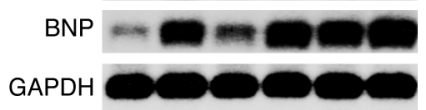

B

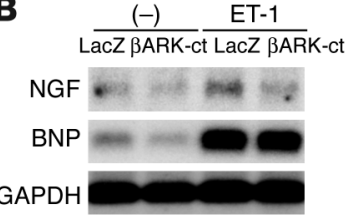

$\mathbf{F}$

$\frac{(-)}{(-)}$

ET-1

NGF

BNP

GAPDH

Relative luciferase activity
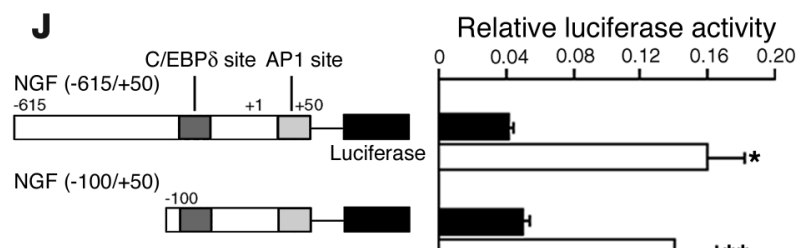

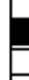

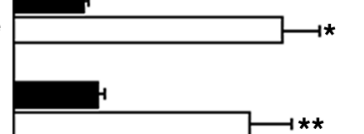

NGF $(-72 /+50)$
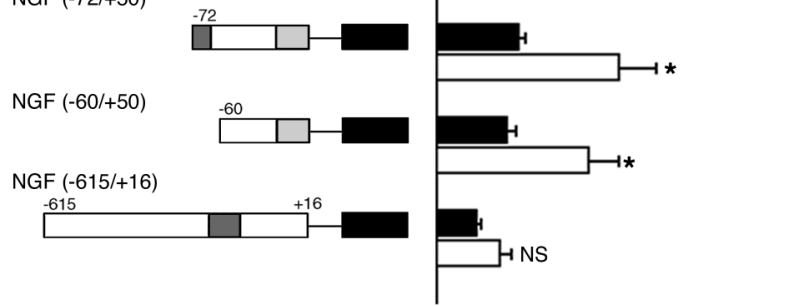

C

(-) ET-1 PMA

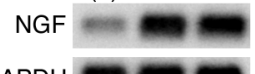

D

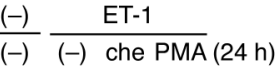

GAPDH

NGF $=0$

GAPDH

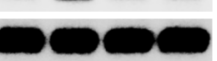

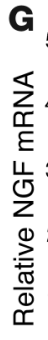

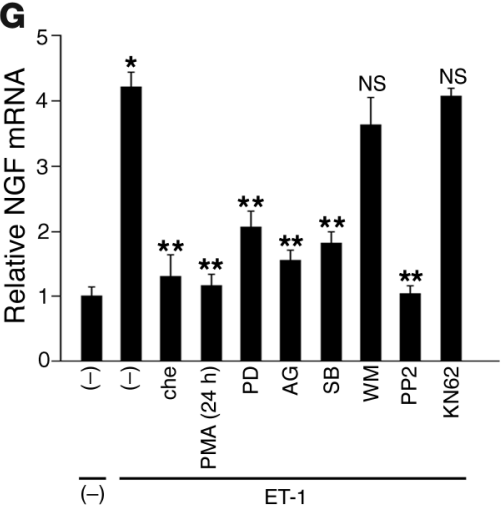

H

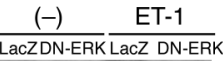

NGF

GAPDH nos thi 1

I

I $\frac{(-)}{\text { LacZ DN-p38 }} \frac{\text { ET-1 }}{\text { LacZ DN-p38 }}$

NGF

GAPDH

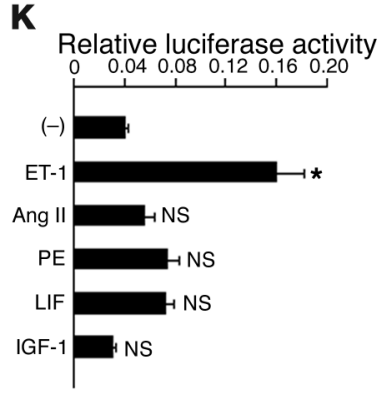

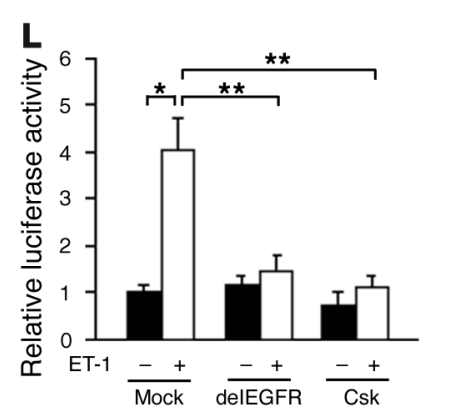

Figure 2

The ET-1/ET receptor augments NGF expression via Gi $\beta \gamma$, PKC, EGFR, ERK, p38MAPK, and AP-1 and C/EBP $\delta$ elements. (A) Preincubation of cardiomyocytes with either PTX or H89. NGF mRNA expression was determined 2 hours after ET-1 stimulation. (B) Cardiomyocytes were pretreated with LacZ or $\beta$ ARK-ct to inhibit the function of Gi $\beta \gamma$, and stimulated with ET-1. $\beta A R K$-ct attenuated ET-1-induced NGF expression, but not BNP. (C and D) Stimulation with PMA (a PKC activator) for 2 hours augmented NGF expression. In contrast, pretreatment with chelerythrine (che; a PKC inhibitor) for 30 minutes or PMA for 24 hours inhibited ET-1-induced NGF expression. (E and F) Pretreatment with PD98059 (PD; an MAPK inhibitor), AG1478 (AG; an EGFR inhibitor), SB203580 (SB; a p38MAPK inhibitor), or PP2 (an Src family inhibitor), but not with wortmannin (WM; a PI3K inhibitor) or KN62 (a calmodulin kinase II/lv inhibitor) attenuated ET-1-induced NGF mRNA expression. BNP was affected only with PD98059 pretreatment. (G) The results of the densitometry of four separate experiments are shown. ${ }^{*} P<0.001$ vs. control; ${ }^{* \star} P<0.01$ vs. ET-1 alone. NS, not significant vs. ET-1 alone. (H and I) Cardiomyocytes were pretreated with DN-ERK or DN-p38MAPK. (J and K) Identification of ET-1-responsive elements in the NGF promoter using luciferase assay. Black bars, control; white bars, ET-1 stimulation $(n=4)$. (L) Specific negative regulatory plasmid of the EGFR (533delEGFR) or the Src family (Csk) inhibited NGF transcription $(n=4)$. ${ }^{*} P<0.001,{ }^{\star *} P<0.01,{ }^{\sharp} P<0.05$ vs. relative control. NS, not significant.

inhibitors, indicating that this pathway is clearly distinct from pathways that mediate hypertrophy. Transfection of DN-ERK or DN-p38 strongly attenuated NGF induction, showing that extracellular signal-regulated kinase (ERK) and p38MAPK are critical in this augmentation (Figure 2, $\mathrm{H}$ and I).

The NGF promoter contains both activator protein-1 (AP-1) and CCAAT/enhancer-binding protein $\delta(\mathrm{C} / \mathrm{EBP} \delta$ ) elements (18). ET-1 augmented luciferase activity from the full-length NGF promoter $(-615 /+50) 4.0$-fold, but deletion of the AP-1 element markedly decreased this augmentation (Figure 2J). The truncation plasmids revealed that the $\mathrm{C} / \mathrm{EBP} \delta$ element was also involved in this induction, but that the AP-1 element was more critical. Other hypertrophic factors did not induce NGF transcription (Figure 2K). Cotransfection of luciferase under the influence of the NGF promoter with a mock plasmid or with the Csk or the 533delEGFR plasmid revealed that Src- and EGFR-mediated signaling was involved in NGF induction (Figure 2L). These results indicate that
Gi $\beta \gamma$, PKC, the Src family, EGFR, ERK, p38MAPK, C/EBP $\delta$, and the AP-1 site are critically involved in this signal transduction pathway.

ET-1 augments NGF-induced differentiation of PC12 cells through NGF secretion from cardiomyocytes. PC12 cells, a rat pheochromocytoma cell line that responds with neurite extension to NGF, was used to assay conditioned medium. To determine whether ET-1-induced NGF production in cardiomyocytes is a biologically relevant phenomenon, we stimulated PC12 cells with conditioned medium for 3 days and examined cell morphology (Figure 3A). Medium conditioned with ET-1-stimulated cardiomyocytes significantly induced neurite outgrowth compared with medium conditioned with unstimulated cardiomyocytes (Figure 3B). Pretreatment of PC12 cells with anti-NGF blocking antibody strongly suppressed cell differentiation (Figure 3C). To mimic sympathetic innervation in the heart, we cocultured PC12 cells with cardiomyocytes and stimulated them with ET-1. PC12 cells showed differentiation that was inhibited by pretreatment with anti-NGF blocking antibody 

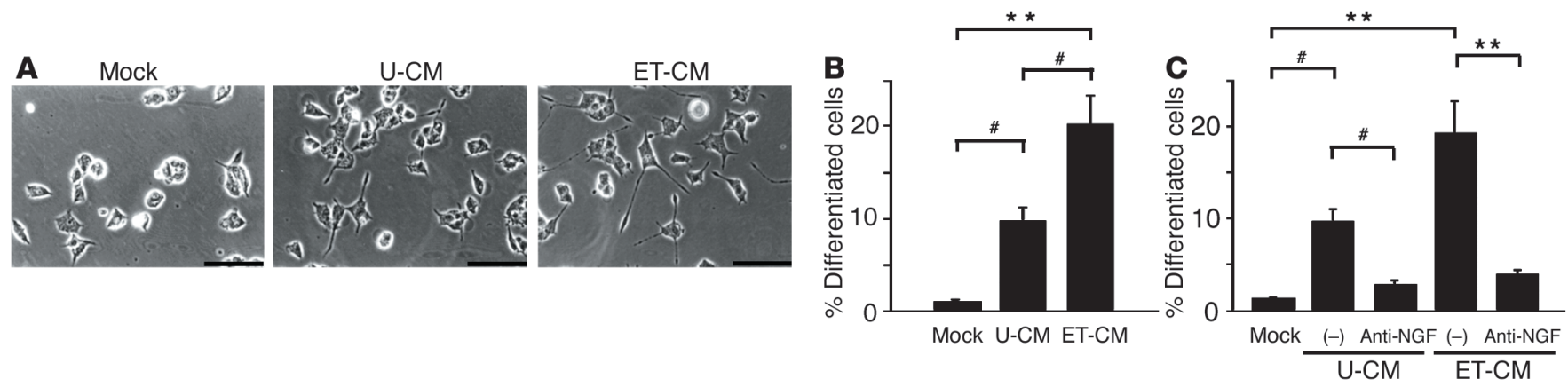

D

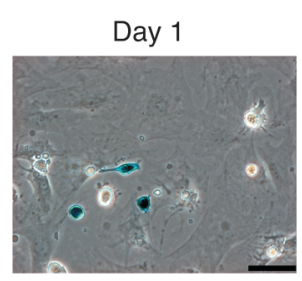

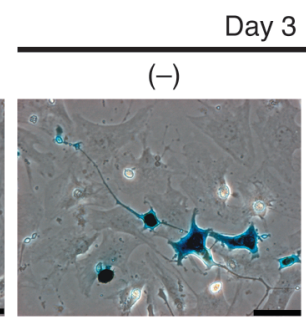

Day $3($ ET-1)

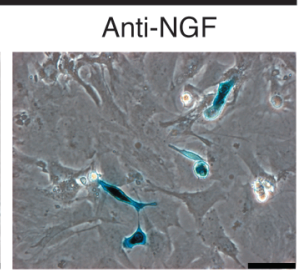

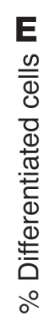
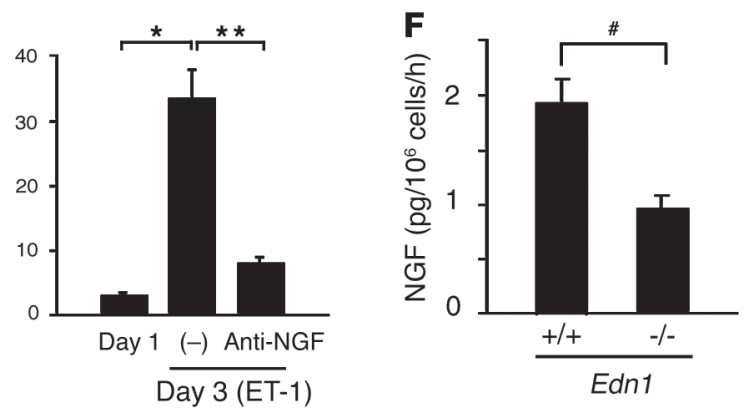

Figure 3

ET-1 causes NGF-mediated differentiation of PC12 cells. (A) PC12 cell morphology was observed after incubation for 3 days in mock medium or medium conditioned with unstimulated cardiomyocytes (U-CM) or ET-1-stimulated cardiomyocytes (ET-CM). Medium conditioned with ET-1-stimulated cardiomyocytes strongly induced neurite extension in PC12 cells compared with medium conditioned with unstimulated cardiomyocytes. (B) Percentage of differentiated cells in A $(n=4)$. (C) PC12 cells were pretreated with anti-NGF blocking antibody for 30 minutes, then incubated with the conditioned medium $(n=4)$. (D) PC12 cells transfected with LacZ were cocultured with cardiomyocytes, then stimulated with ET-1 or ET-1 plus anti-NGF blocking antibody for 3 days. PC12 cells were identified using X-gal staining. (E) Percentage of differentiated cells in $\mathbf{D}(n=4)$. (F) NGF protein levels in medium conditioned with Edn1+/+ or Edn1-/- cardiomyocytes were measured by ELISA $(n=4) .{ }^{*} P<0.0001 ;{ }^{* \star} P<0.001 ;{ }^{\#} P<0.01$. Scale bar: $100 \mu$ m.

(Figure 3, D and E), indicating that the NGF secreted by cardiomyocytes is capable of stimulating neuritogenesis in PC12 cells.

To investigate whether endogenous ET-1 secreted by cardiomyocytes is critical for NGF production, we measured the NGF protein level in medium conditioned with $E d n 1^{-/-}$or $E d n 1^{+/+}$cardiomyocytes and found it to be reduced by half in medium conditioned with Edn1 $1^{-/-}$cardiomyocytes (Figure 3F).

Disruption of ET-1, but not angiotensinogen, reduces NGF expression, sympathetic nerve density, and norepinephrine concentration in the heart. To investigate whether ET-1-specific induction of NGF in cardiomyocytes participated in cardiac sympathetic nerve development, we analyzed $E d n 1^{-/-}$mouse hearts at embryonic day (E) 18.5. $\mathrm{Atg}^{-/}$mice were used as a control. The levels of NGF mRNA in $E d n 1^{-/-}$mouse hearts were downregulated to $32 \%$ of those in $E d n 1^{+/+}$hearts (Figure 4A), while those in $\mathrm{Atg}^{-1-}$ mice showed no change (97\% of those in $\mathrm{Atg}^{+/+}$mice). The mRNA levels of neurotrophin-3, another neurotrophic factor known to induce sympathetic innervation (28), were unaffected in $\mathrm{EdnI}^{-/_{-}}$and $\mathrm{Atg}^{/-}$mice (Figure 4B).

To determine the cardiac sympathetic nerve density in these mice, immunostaining was conducted using antibodies to $\mathrm{TH}$, a marker for sympathetic nerves, GAP43, a marker for nerve sprouting, and PGP9.5, a general marker for peripheral neurons. Immunostaining for TH, GAP43, and PGP9.5 was performed on serial sections and labeled the same structures in several areas of the heart (data not shown), as described previously $(7,8)$. At E18.5, most sympathetic nerve endings were restricted to the epicardium in both $E d n 1^{-/-}$and $\mathrm{Atg}^{-1-}$ mice. Surprisingly, GAP43, PGP9.5, and TH immunoreactivities were markedly decreased only in $E d n 1^{-/-}$mice (Figure 4, C-F).
The concentration of total cardiac norepinephrine was significantly lower in $E d n 1^{-/-}$mice than in $E d n 1^{+/+}$littermates but was unaffected in $\mathrm{Atg}^{-1-}$ mice (Figure 4G). These results indicated that NGF expression and cardiac sympathetic innervation are specifically reduced in $E d n 1^{-/-}$hearts.

ET-1-deficient mice display a loss of SG neurons by apoptosis during periods of NGF dependence. The cardiac sympathetic nerve extends from the sympathetic neurons in SG, which are derived from neural crest cells. Neural crest cells migrate and form sympathetic ganglia by E11.5, then proliferate and differentiate into mature neurons. To examine whether ET-1 affects the early stage of sympathetic trunk formation in SG, we immunostained whole-mount $E d n 1^{-/-}$embryos with antiTH antibody at E12.5 (Figure 5A). $\mathrm{TH}^{+}$neurons formed normal ganglia and sympathetic trunks bilaterally to the vertebra. Next, we examined the size and cellularity of SG by cresyl violet staining and immunostaining for TH (Figure 5, B, C, and F). At E12.5 and E15.5, neuronal cell counts and area were unaffected in $E d n 1^{-/}$SG compared with WT. Moreover, TH immunoreactivities were not changed in $E d n 1^{-/-}$SG, indicating that migration and differentiation of neural crest cells in SG were not disrupted in the early stages. At E18.5, however, $E d n 1^{-/-}$SG were markedly smaller than those found in WT embryos, contained fewer neurons (55\% of WT), and had a mean neuronal area that was $73 \%$ smaller than that of WT embryos. Thus, Edn1 $1^{-1-}$ SG exhibited a dramatic loss of sympathetic neurons between E15.5 and E18.5. To examine the cause of the loss of SG neurons in $E d n 1^{-/}$embryos, sections were processed with Ki-67 immunostaining to assess the level of proliferation (Figure 5, D and G). Edn1 $1^{-/-}$SG displayed nearly identical levels of proliferation compared with WT at 
A
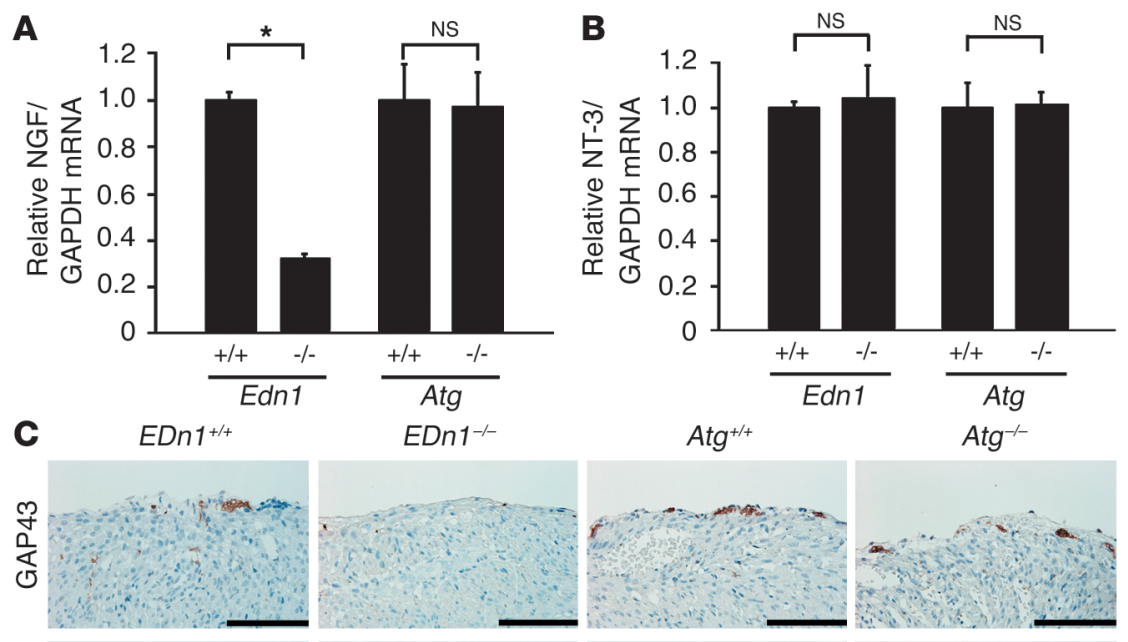
$E D n 1^{-1-}$
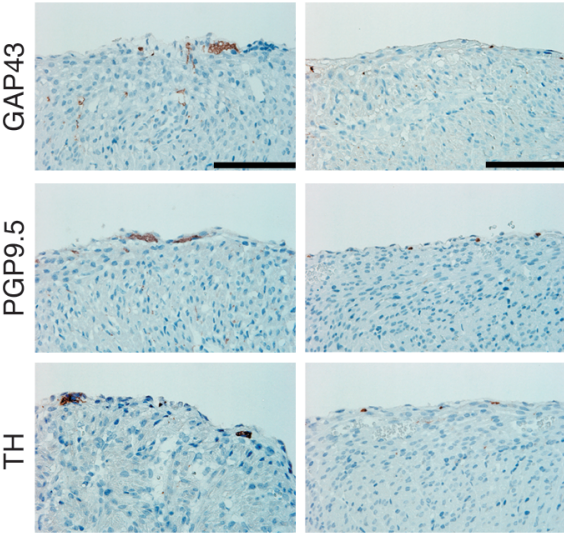

D

$\mathbf{F}$

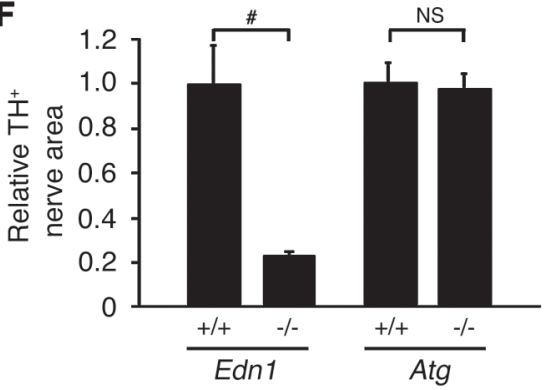

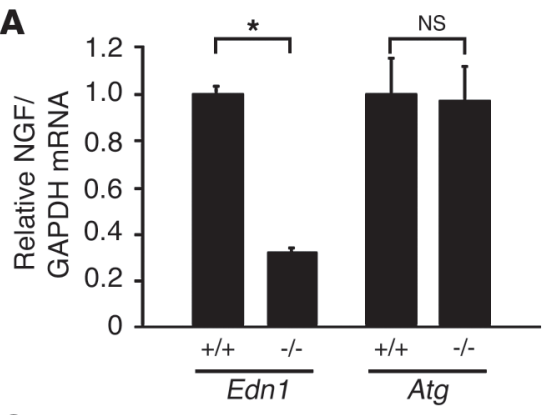

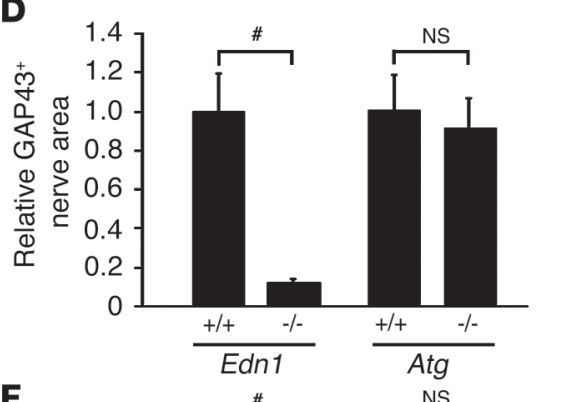
$\operatorname{Atg}^{+/+}$

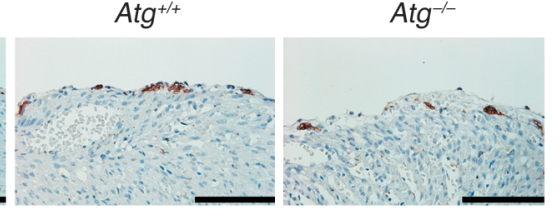

Atg
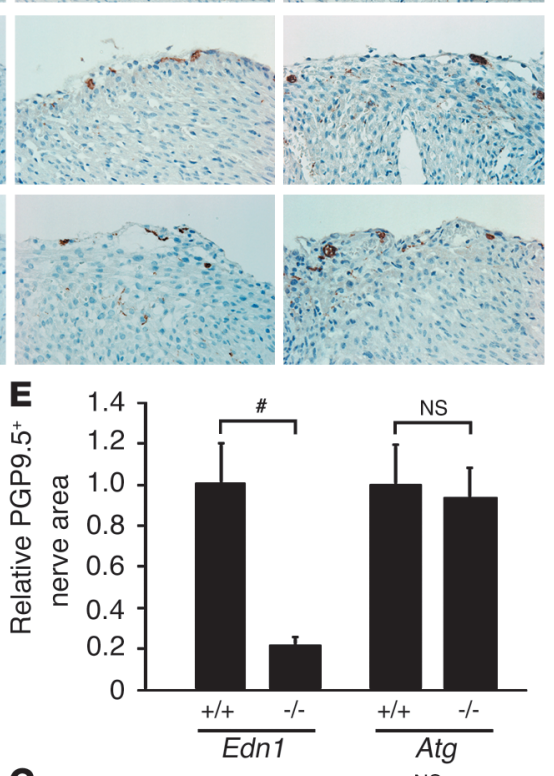

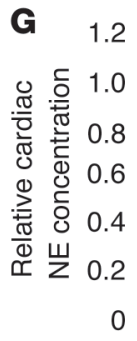
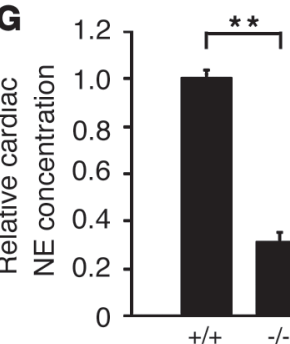

Edn1
Figure 4

Disruption of ET-1, but not of angiotensinogen, reduces NGF expression, sympathetic nerve density, and norepinephrine concentration in murine

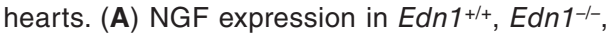
$\mathrm{Atg}^{+/+}$, and $\mathrm{Atg}^{-/-}$hearts at E18.5 was determined by quantitative RT-PCR $(n=10)$. (B) Neurotrophin3 (NT-3) expression in the heart was measured by quantitative RT-PCR. The same reverse transcription products used in A were analyzed. $(n=10$.) (C) Immunostaining for GAP43, PGP9.5, and TH in the heart. Nerves were restricted to the epicardium in both genotypes, and levels of GAP43, PGP9.5, and TH were lower in Edn1-/- mice, but not in $\mathrm{Atg}^{-/-}$mice, compared with WT littermates. (D-F) The immunopositive nerve areas for GAP43, PGP9.5, and TH were determined using NIH Image $(n=8)$. (G) Cardiac norepinephrine (NE) concentrations were measured by HPLC $(n=10) .{ }^{*} P<0.0001 ;{ }^{* *} P<0.005 ;{ }^{*} P<0.01$. NS, not significant. Scale bar: $100 \mu \mathrm{m}$.

detected from E12.5 and increased sequentially, but levels were similar in $E d n 1^{-/-}$and $E d n 1^{+/-}$ hearts at the early stages. Sympathetic nerve endings were not detected at E12.5 (data not shown) and appeared from E15.5, but no difference was observed at this stage. These findings suggested that sympathetic nerve fibers initially reached the heart and that disruption of subsequent innervation was coincident with NGF downregulation in $E d n 1^{-/-}$hearts between E15.5 and E18.5.

Cardiac-specific overexpression of NGF overcomes the defects of the cardiac sympathetic nervous system in Edn1 $1^{-/-}$mice. It is possible that the low sympathetic nerve density in the heart and excess apoptosis in SG neurons in $E d n 1^{-/-}$mice are a direct effect of ET-1 deficiency. To address this question, we initiated a genetic rescue of cardiac NGF expression in $E d n 1^{-/-}$mice to investigate whether the defects were caused by reduced NGF expression. Transgenic mice overexpressing rat NGF under the control of $\alpha$-myosin heavy chain promoter were bred onto the $E d n 1^{+/}$- background to restore NGF activity specifically to the hearts of Edn 1-/- embryos. Edn 1-/-/MHC-NGF mice died postnatally and had craniofacial defects similar to those observed in $E d n 1^{-/-}$mice. Quantitative RT-PCR revealed that strong NGF expression, 14.5-fold that of the control mice, was detected each stage. Given that the requirement of NGF for the survival of sympathetic neurons begins at E16.5, we used TUNEL staining to address whether excess apoptosis accounts for the loss of neurons. At E12.5 and E15.5, excess apoptosis was not detected, but at E18.5, $E d n 1^{-/-} \mathrm{SG}$ displayed a fourfold increase in the number of TUNEL ${ }^{+}$cells compared with those of WT littermates (Figure 5, E and H). These results indicated that loss of sympathetic SG neurons in $E d n 1^{-/-}$mice results from excess neuronal apoptosis in late gestation but not from a failure in neuronal migration, differentiation, or proliferation.

Next, we analyzed the time course of cardiac innervation and NGF levels in $E d n 1^{-/-}$and $E d n 1^{+/+}$hearts (Figure 5, I-K). NGF mRNA was in $E d n 1^{-/-} / \mathrm{MHC}-\mathrm{NGF}$ hearts at E18.5 (Figure 6A). Immunostaining for TH (Figure 6, B and C), GAP43, and PGP9.5 (data not shown) showed that $E d n 1^{-/-} / \mathrm{MHC}-\mathrm{NGF}$ mice had hyperinnervation compared with $E d n 1^{-/-}$littermates. The norepinephrine concentration was markedly increased in Edn1 $1^{-/-} / \mathrm{MHC}-\mathrm{NGF}$ hearts (Figure 6D). These results show that cardiac-specific NGF overexpression restores sympathetic nerve density in $E d n 1^{-/-}$hearts. Next, $E d n 1^{-/-} / \mathrm{MHC}-\mathrm{NGF}$ SG were examined by cresyl violet staining, immunostaining for $\mathrm{TH}$ and Ki-67, and TUNEL staining at E18.5 (Figure 6, E-G). Loss of sympathetic neurons and reduced neuronal area were completely overcome, and the level of TUNEL ${ }^{+}$cells also decreased. These results sup- 
A
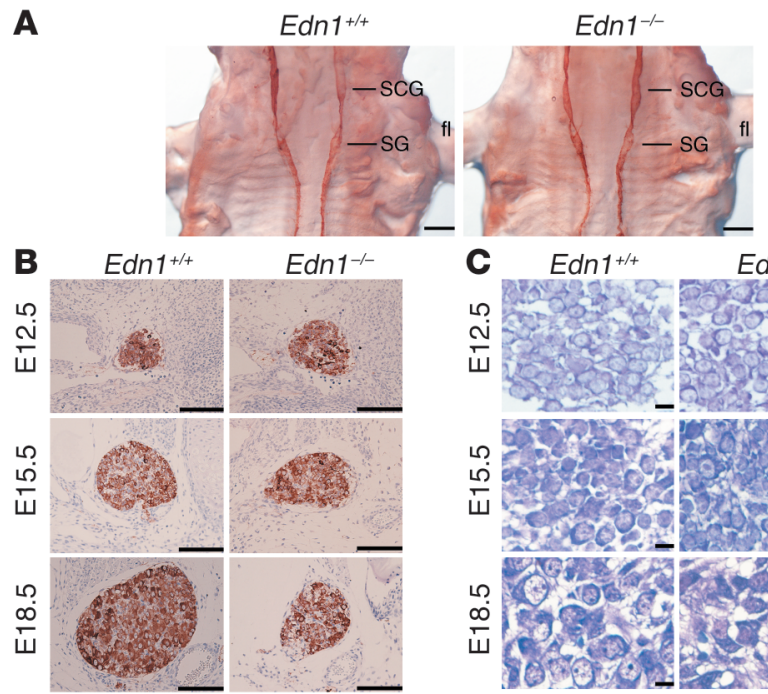

$\mathbf{F}$
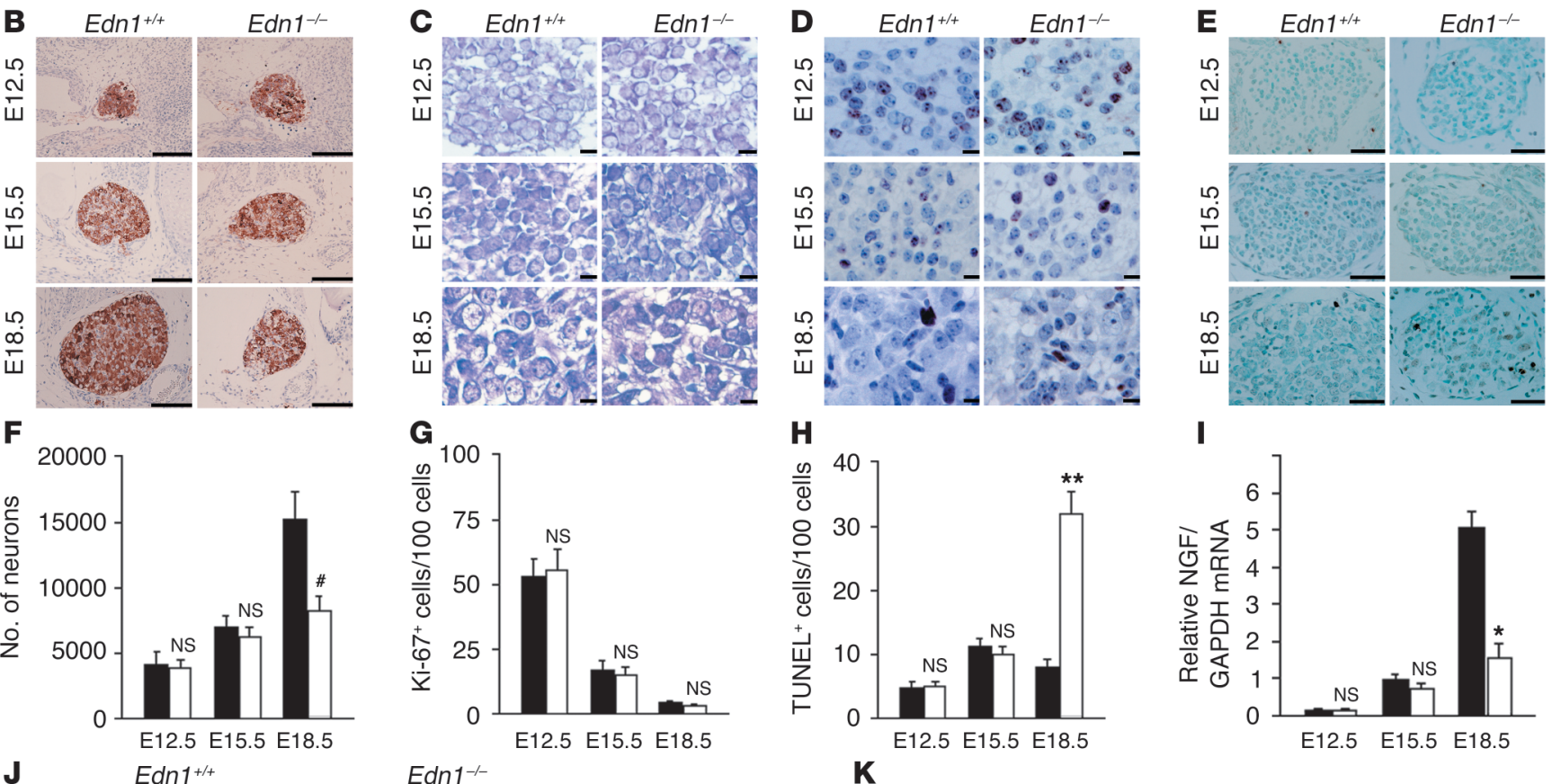

\section{H}

\section{I}
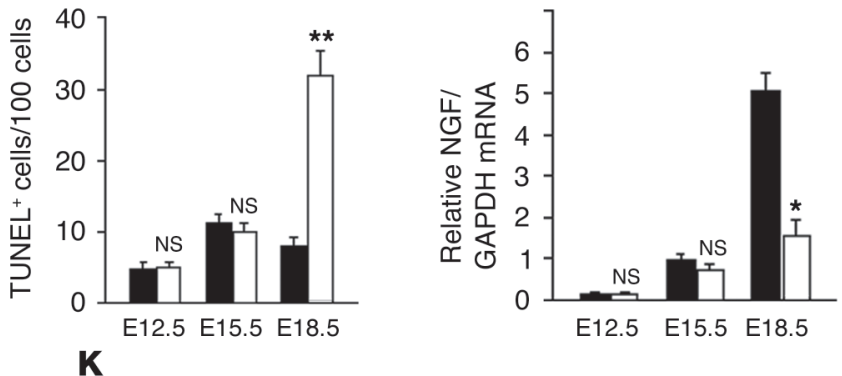

Edn1-1-

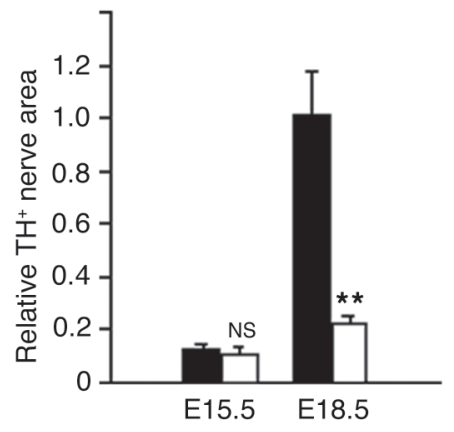

\section{Figure 5}

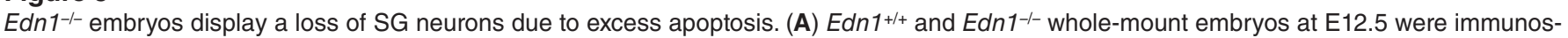
tained with anti-TH antibody. SCG, superior cervical ganglion; fl, forelimb. Similar results were obtained from four separate experiments. (B-E) TH

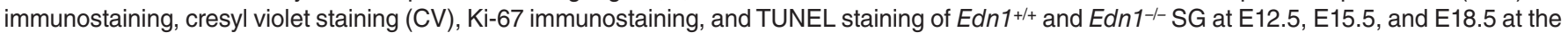

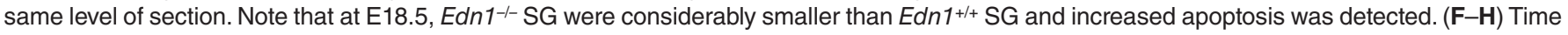
course of the number of neurons, Ki-67+ cells per 100 neurons, and TUNEL+ cells per 1,000 neurons in SG was shown $(n=5)$. (I) Time course of NGF expression in $E d n 1^{+/+}$and $E d n 1^{-/-}$hearts was determined by quantitative RT-PCR $(n=3)$. ( $\mathbf{J}$ and $\left.\mathbf{K}\right)$ Immunostaining for TH in the heart of Edn $1^{+/+}$and Edn1 $1^{-/}$embryos at E15.5 and E18.5. TH-immunopositive nerve fibers were slightly detected from E15.5. LA, left atrium; LV, left ventricle. The immunopositive nerve areas for TH were determined using NIH Image. $(n=4$. $){ }^{*} P<0.001 ;{ }^{*} P<0.01 ;{ }^{*} P<0.05$. NS, not significant vs. relative control. Scale bar: $500 \mu \mathrm{m}$ (A), $100 \mu \mathrm{m}$ (B and J), $10 \mu \mathrm{m}$ (C and D), $50 \mu \mathrm{m}$ (E). Black bars, Edn1 ${ }^{+/+}$; white bars, Edn1 $1^{-/-}$.

port the hypothesis that the ET-1-NGF pathway plays a critical role in development of the cardiac sympathetic nervous system.

\section{Discussion}

Cardiac performance is tightly controlled by the autonomic nervous system. NGF is the best-characterized and most well-known member of the neurotrophin family, which contributes to the development and maintenance of sympathetic innervation. The level of NGF synthesized in the target organ determines its innervation density (2). However, the molecular mechanisms that regulate NGF expression and sympathetic innervation remain poorly understood. In this study, we found that (a) ET-1, but not angiotensin II, phenylephrine, LIF, or IGF-1, induces NGF augmentation in cardiomyocytes; (b) ET-1-induced NGF augmentation is mediated by the $\mathrm{ET}_{\mathrm{A}}$ receptor, $\mathrm{Gi} \beta \gamma$, $\mathrm{PKC}$, the Src family, 
A

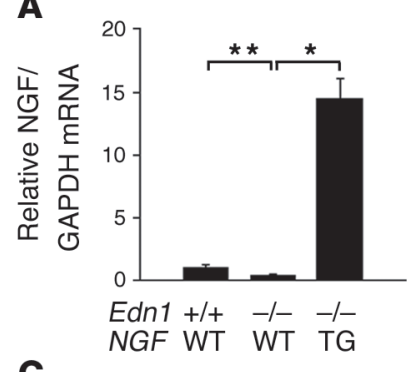

C
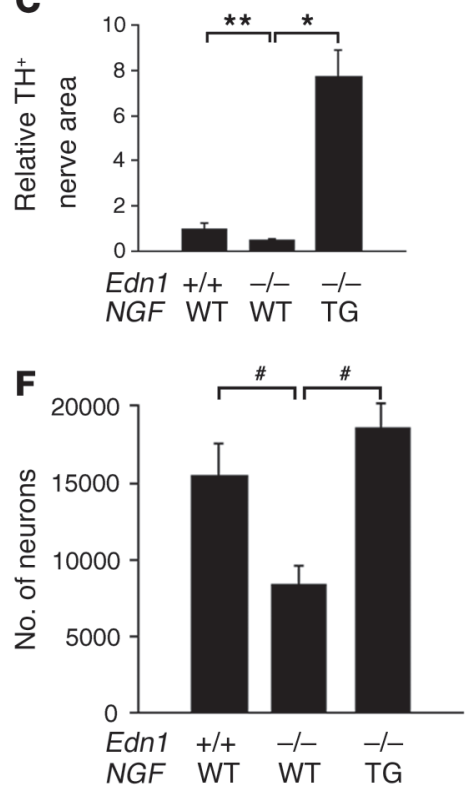

B $\underset{N G F}{E d n 1}$
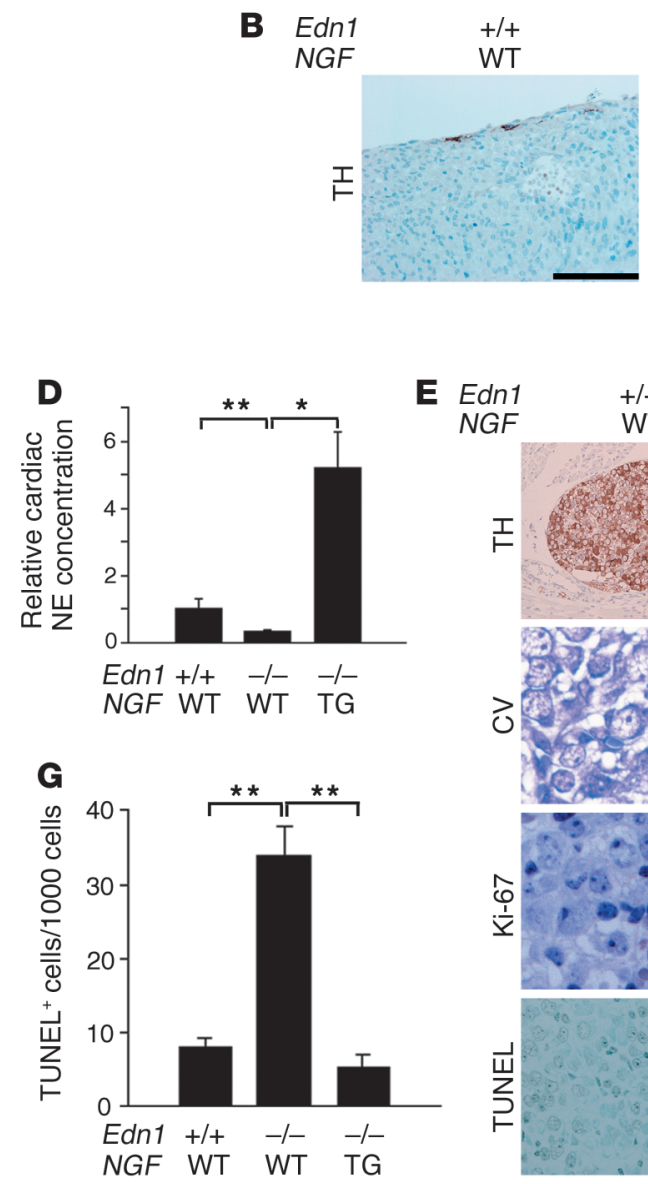

E $\begin{aligned} & E d n 1 \\ & N G F\end{aligned}$

$\begin{array}{ll}\text { NGF } & +/+ \\ & \text { WT }\end{array}$
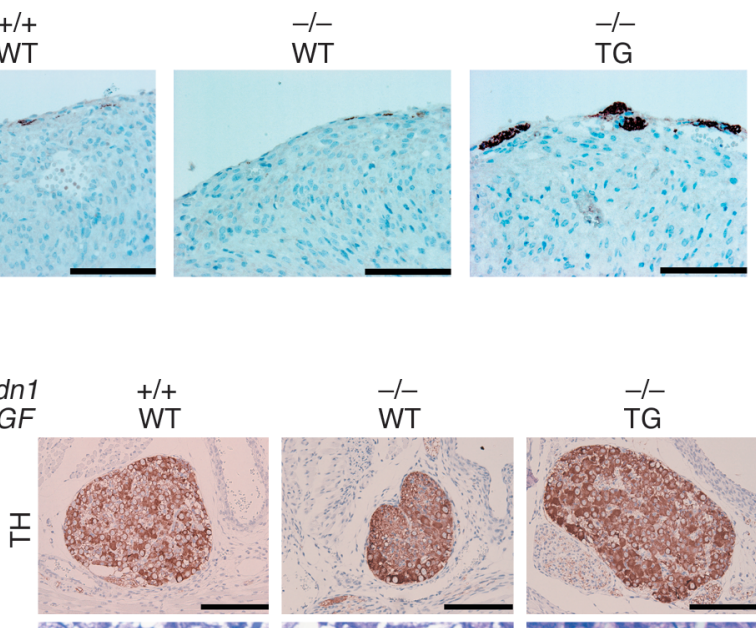

$\stackrel{-l-}{\mathrm{TG}}$

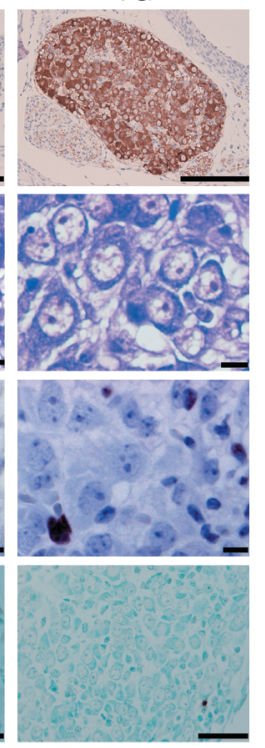

\section{Figure 6}

Cardiac-specific overexpression of NGF overcomes the defects of the cardiac sympathetic nervous system in Edn1 $1^{-/-}$mice. (A) NGF expression in Edn1 $1^{+/+}$,

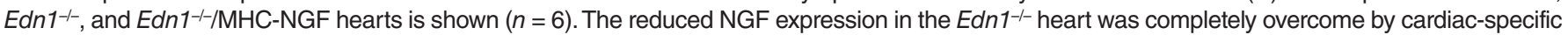

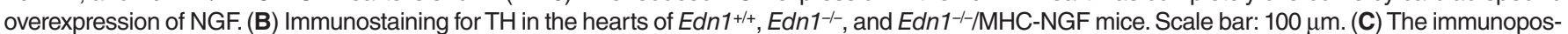
itive nerve areas for TH were quantitated $(n=6)$. (D) The cardiac norepinephrine (NE) concentration was increased in Edn1-l-/MHC-NGF mice compared

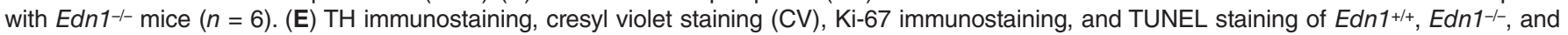
Edn1-1-/MHC-NGF SG at E18.5 at the same level of section. Note that the reduction of the size of SG and the increase in TUNEL+ cells in Edn1 ${ }^{-1-}$ mice were completely reversed in Edn1--/MHC-NGF mice. (F and $\mathbf{G})$ The number of neurons and the number of TUNEL ${ }^{+}$cells per 1,000 neurons in each SG are shown $(n=3-6) .{ }^{*} P<0.0001 ;{ }^{* \star} P<0.01 ;{ }^{*} P<0.05$. TG, transgenic. Scale bar: $100 \mu \mathrm{m}(\mathrm{TH}), 50 \mu \mathrm{m}(\mathrm{TUNEL}), 10 \mu \mathrm{m}(\mathrm{CV}$ and Ki-67).

EGFR, ERK, and P38MAPK, but not by PKA, PI3K, or calmodulin kinase II or IV; (c) AP-1 and C/EBP $\delta$ elements are essential cis-elements for ET-1-induced NGF transcription, with AP-1 being the more critical of the two; (d) ET-1-induced NGF augmentation in cardiomyocytes stimulates the differentiation of PC12 cells; and (e) the analysis of $E d n 1^{-/-}$and Edn1 $1^{-/} /$MHC-NGF mice demonstrated that ET-1 is required for induction of NGF expression and for promotion of sympathetic innervation and survival of SG neurons. These results show that ET-1-specific regulation of NGF in cardiomyocytes plays a critical role in the development of the cardiac sympathetic nervous system.

Of the cardiac hypertrophic factors investigated in this study, only ET-1 augmented NGF expression, and the ET-1-NGF pathway was mediated by the $\mathrm{ET}_{\mathrm{A}}$ receptor and $\mathrm{Gi} \beta \gamma$. The $\mathrm{ET}_{\mathrm{A}}$ receptor is known to activate Gs, Gq, and Gi proteins, while phenylephrine and angiotensin II activate Gq and Gs proteins (29). The characteristic coupling of the $\mathrm{ET}_{\mathrm{A}}$ receptor to $\mathrm{G}$ protein subunits might explain the specificity of ET-1-induced NGF augmentation. In contrast to the present study, a previous study reported that the cAMP-PKA pathway was involved in $\beta$-adrenoreceptor-mediated NGF augmentation in astrocytoma cells (30). The finding that ET-1 does not induce NGF augmentation in cardiac fibroblasts suggests that the NGF induction pathway is mediated in a cell type-specific manner.

In this study, we found that the differentiation of PC12 cells was enhanced when the cultures contained medium conditioned with ET-1-stimulated cardiomyocytes or were cocultured with cardiomyocytes treated with ET-1. Our results point to a critical role for ET-1-induced NGF production in the promotion of neurite extension. This is based on our finding that ET-1-induced neurite extension is completely blocked by anti-NGF antibody, and that ET-1 alone did not augment PC12 cell differentiation (data not shown). The blocking, by anti-NGF blocking antibody, of neurite extension induced by medium conditioned with unstimulated cardiomyocytes shows that cardiomyocytes secrete a basal amount of NGF.

Experimental sympathectomy does not alter the onset or extent of NGF mRNA accumulation in target organs, which indicates that regulation of NGF synthesis during development is independent of inner- 
vation or norepinephrine secreted from sympathetic nerves (31). Upstream molecules that regulate NGF expression in vivo remain undetermined. The present study demonstrated that NGF was downregulated and sympathetic innervation in the heart was reduced in $\mathrm{Edn1^{-/- }}$ mice, but not in $\mathrm{Atg} \mathrm{g}^{-/-}$mice. Moreover, in $\mathrm{Edn1^{-/- }}$ mice, SG that contribute to the sympathetic innervation of the heart revealed neuronal loss due to excess apoptosis at the late embryonic stage, but not due to failure in neuronal migration, differentiation, or proliferation. These findings are consistent with previous reports that increased pyknosis is detected from E16.5 in NGF-targeted mice and that NGF transported from target organs acts on survival of innervating neurons, but not on proliferation or differentiation of sympathetic neurons (24). Developing axons are guided to their targets and maintained by extracellular molecules. Neurotrophin-3 is also a critical factor for the survival and differentiation of sympathetic neurons $(2,5,28)$. However, neurotrophin-3 was not downregulated in $E d n 1^{-/-}$heart, and the sympathetic neuronal defects revealed in $E d n 1^{-/-}$mice were restored by overexpression of NGF in the heart. Taken together, these findings suggest that ET-1 is a key regulator of NGF induction in the heart and plays a specific and critical role in construction of the cardiac sympathetic nervous system via the regulation of NGF production. To our knowledge, this is the first report to identify a molecule that regulates NGF production in sympathetic target organs.

From a clinical perspective, $\mathrm{ET}_{\mathrm{A}}$ receptor antagonists are known to improve the prognosis of heart failure by preventing cardiac remodeling and ventricular dysfunction (32). $\mathrm{ET}_{\mathrm{A}}$ receptor antagonists also have an antiarrhythmic effect in pathological hearts, although the mechanism remains unclear (33). Neural remodel- ing in sympathetic nerve sprouting results in ventricular tachyarrhythmia in diseased human hearts and in animal models $(7,8)$. In contrast, $\beta$-blocker therapy decreases the risk of sudden death secondary to ventricular tachyarrhythmia in ischemic heart disease or congestive heart failure. Given that ET-1 is strongly induced in the process of heart disease, the beneficial effects of $\mathrm{ET}_{\mathrm{A}}$ receptor antagonists as antiarrhythmic agents may be related to the remodeling of the sympathetic nervous system that is mediated by the ET-1-NGF pathway. Further studies are needed to investigate whether ET-1 augmentation leads to an increase in NGF in the diseased heart.

In conclusion, these findings indicate that ET-1 regulates NGF expression in cardiomyocytes and plays a critical role in sympathetic innervation of the heart.

\section{Acknowledgments}

This study was supported in part by research grants from the Ministry of Education, Culture, Sports, Science and Technology, Japan, and Health Science Research Grants for Advanced Medical Technology from the Ministry of Health, Labor and Welfare, Japan.

Received for publication July 14, 2003, and accepted in revised form December 16, 2003.

Address correspondence to: Keiichi Fukuda, Institute for Advanced Cardiac Therapeutics, Keio University School of Medicine, 35 Shinanomachi, Shinjuku-ku, Tokyo 160-8582, Japan. Phone: 81-35363-3874; Fax: 81-3-5363-3875; E-mail: kfukuda@sc.itc.keio.ac.jp.
1. Loring, J.F., and Erickson, C.A. 1987. Neural crest cell migratory pathways in the trunk of the chick embryo. Dev. Biol. 121:220-236.

2. Snider, W.D. 1994. Functions of the neurotrophins during nervous system development: what the knockouts are teaching us. Cell. 77:627-638.

3. Lockhart, S.T., Turrigiano, G.G., and Birren, S.J. 1997. Nerve growth factor modulates synaptic transmission between sympathetic neurons and cardiac myocytes. J. Neurosci. 17:9573-9582.

4. Heumann, R., Korsching, S., Scott, J., and Thoenen, H. 1984. Relationship between levels of nerve growth factor (NGF) and its messenger RNA in sympathetic ganglia and peripheral target tissues. EMBO J. 3:3183-3189.

5. Brennan, C., Rivas-Plata, K., and Landis, S.C. 1999. The p75 neurotrophin receptor influences NT-3 responsiveness of sympathetic neurons in vivo. Nat. Neurosci. 2:699-705.

6. Hassankhani, A., et al. 1995. Overexpression of NGF within the heart of transgenic mice causes hyperinnervation, cardiac enlargement, and hyperplasia of ectopic cells. Dev. Biol. 169:309-321.

7. Cao, J.M., et al. 2000. Relationship between regional cardiac hyperinnervation and ventricular arrhythmia. Circulation. 101:1960-1969.

8. Cao, J.M., et al. 2000. Nerve sprouting and sudden cardiac death. Circ. Res. 86:816-821.

9. Kanki, H., et al. 1999. Comparison of nerve growth factor mRNA expression in cardiac and skeletal muscle in streptozotocin-induced diabetic mice. Life Sci. 65:2305-2313.

10. Kaye, D.M., Vaddadi, G., Gruskin, S.L., Du, X.J., and Esler, M.D. 2000. Reduced myocardial nerve growth factor expression in human and experimental heart failure. Circ. Res. 86:E80-E84.

11. Kurihara, Y., et al. 1994. Elevated blood pressure and craniofacial abnormalities in mice deficient in endothelin-1. Nature. 368:703-710.
12. Kurihara, Y., et al. 1995. Aortic arch malformations and ventricular septal defect in mice deficient in endothelin-1. J. Clin. Invest. 96:293-300.

13. Clouthier, D.E., et al. 1998. Cranial and cardiac neural crest defects in endothelin-A receptor-deficient mice. Development. 125:813-824.

14. Sano, M., et al. 2000. Interleukin- 6 family of cytokines mediate angiotensin II-induced cardiac hypertrophy in rodent cardiomyocytes. J. Biol. Chem. 275:29717-29723.

15. Akamatsu, W., et al. 1999. Mammalian ELAV-like neuronal RNA-binding proteins $\mathrm{HuB}$ and $\mathrm{HuC}$ promote neuronal development in both the central and the peripheral nervous systems. Proc. Natl. Acad. Sci. U. S. A. 96:9885-9890.

16. Nishida, M., et al. 2000. G alpha (i) and G alpha (o) are target proteins of reactive oxygen species. Nature. 408:492-495.

17. Zhan, Y., et al. 2003. Role of JNK, p38, and ERK in platelet-derived growth factor-induced vascular proliferation, migration, and gene expression. Arterioscler. Thromb. Vasc. Biol. 23:795-801.

18. Colangelo, A.M., Johnson, P.F., and Mocchetti, I. 1998. Beta-adrenergic receptor-induced activation of nerve growth factor gene transcription in rat cerebral cortex involves CCAAT/enhancer-binding protein delta. Proc. Natl. Acad. Sci. U. S. A. 95:10920-10925.

19. Kodama, H., et al. 2003. Selective involvement of p130Cas/Crk/Pyk2/c-Src in endothelin-1-induced JNK activation. Hypertension. 41:1372-1379.

20. Tanimoto, K., et al. 1994. Angiotensinogen-deficient mice with hypotension. J. Biol. Chem. 269:31334-31337.

21. Nishimura, Y., Ito, T., Hoe, K., and Saavedra, J.M. 2000. Chronic peripheral administration of the angiotensin II AT(1) receptor antagonist candesartan blocks brain $\mathrm{AT}(1)$ receptors. Brain Res. 871:29-38.

22. Hjemdahl, P. 1984. Catecholamine measurements by high-performance liquid chromatography. Am. J. Physiol. 247:E13-E20.
23. Kawasaki, T., et al. 2002. Requirement of neuropilin 1mediated Sema3A signals in patterning of the sympathetic nervous system. Development. 129:671-680.

24. Francis, N., et al. 1999. NT-3, like NGF, is required for survival of sympathetic neurons, but not their precursors. Dev. Biol. 210:411-427.

25. Selby, M.J., Edwards, R., Sharp, F., and Rutter, W.J. 1987. Mouse nerve growth factor gene: structurea and expression. Mol. Cell. Biol. 7:3057-3064.

26. Chiloeches, A., et al. 1999. Regulation of Ras. GTP loading and Ras-Raf association in neonatal rat ventricular myocytes by $\mathrm{G}$ protein-coupled receptor agonists and phorbol ester. Activation of the extracellular signal-regulated kinase cascade by phorbol ester is mediated by Ras. J. Biol. Chem. 274:19762-19770.

27. Sugden, P.H., and Clerk, A. 1997. Regulation of the ERK subgroup of MAP kinase cascades through G protein-coupled receptors. Cell. Signal. 9:337-351.

28. Story, G.M., et al. 2000. Inactivation of one copy of the mouse neurotrophin-3 gene induces cardiac sympathetic deficits. Physiol. Genomics. 27:129-136.

29. Zou, Y., et al. 1998. Cell type-specific angiotensin IIevoked signal transduction pathways. Circ. Res. 82:337-345.

30. Mocchetti, I., et al. 1989. Regulation of nerve growth factor biosynthesis by beta-adrenergic receptor activation in astrocytoma cells: a potential role of c-Fos protein. Proc. Natl. Acad. Sci. U. S. A. 86:3891-3895.

31. Clegg, D.O., Large, T.H., Bodary, S.C., and Reichardt, L.F. 1989. Regulation of nerve growth factor mRNA levels in developing rat heart ventricle is not altered by sympathectomy. Dev. Biol. 134:30-37.

32. Sakai, S., et al. 1996. Inhibition of myocardial endothelin pathway improves long-term survival in heart failure. Nature. 384:353-355.

33. Matsumoto, Y., et al. 2002. Long-term endothelin a receptor blockade inhibits electrical remodeling in cardiomyopathic hamsters. Circulation. 106:613-619. 